International Mathematical Forum, Vol. 9, 2014, no. 6, 255 - 258

HIKARI Ltd, www.m-hikari.com

http://dx.doi.org/10.12988/imf.2014.413

\title{
A Generalization of Weakly Co-hopfian Modules
}

\author{
Xu Wang \\ School of Science, Lanzhou University of Technology \\ 287, Langongping Road, Qilihe Area, Lanzhou \\ Gansu, 730050, P.R. China \\ Tingting Li \\ College of Science, Guilin University of Technology \\ 12, Jiangan Road, Qixing Area, Guilin \\ Guangxi, 451004, China
}

Copyright (C) $2014 \mathrm{Xu}$ Wang and Tingting Li. This is an open access article distributed under the Creative Commons Attribution License, which permits unrestricted use, distribution, and reproduction in any medium, provided the original work is properly cited.

\begin{abstract}
An R-module $M$ is called t-weakly co-hopfian if any monomorphic endomorphism of $M$ is t-essential, which is discussed in [3]; still its property much familiar with weakly co-hopfian class, though, $M$ is different due to its non-zero $Z_{2}(M)$. Several equivalent conditions are given for a module to be t-weakly co-hopfian.
\end{abstract}

Mathematics Subject Classification: 16D10

Keywords: t-weakly co-hopfian, weakly co-hopfian

\section{Introduction}

Rings will have unit elements and modules will be unitary. The terminology not defined here may be found in [1]. Let $R$ be a ring and $M_{R}$ a right $R$-module. $M$ is called co-hopfian if any injective endomorphism of $M$ is an isomorphism, and called weakly co-hopfian if any injective endomorphism of $M$ is essential; see[2] for a discussion of such modules. We say an $R$-module $M$ is t-weakly co-hopfian if any injective endomorphism $f$ of $M$ is t-essential; that is, for any submodules $N$ of $M, f(M) \cap N \leq_{\text {tes }} Z_{2}(M)$ implies that $N \leq Z_{2}(M)$. 


\section{Main Results}

Lemma 2.1 Let $A, B$ be submodules of $M, A \leq B$, if $A$ is t-essential in $M$, then also $B$.

Proof: If $B$ is not t-essential in $M$, then there exist $S \leq M$ satisfies $S \cap B \leq Z_{2}(M), S$ is not in $Z_{2}(M)$. Because $A \leq B$, then $S \leq M$ satisfies $S \cap A \leq Z_{2}(M)$, $S$ is not in $Z_{2}(M)$, contradicts.

Theorem 2.2 The followings are equivalent conditions on a right $R$-module $M$.

(1) $M_{R}$ is t-weakly co-hopfian. (2) For any right $R$-module $N$, if there is an $R$-monomorphism $f: M \oplus N \rightarrow M$, then $f(N) \leq Z_{2}(M)$; that is, $N$ is $Z_{2}$-torsion.

(2') For any right $R$-module $N$, if $f: M \oplus N \rightarrow M$ is a t-essential monomorphism, then $f(N) \leq Z_{2}(M)$; that is, $N$ is $Z_{2}$-torsion.

(3) $M^{\prime}=M / Z_{2}(M)$, then $M^{\prime}$ is weakly co-hopfian(Dedekind finite).

(4) There exists a fully invariant ( $t$-) essential submodule which is t-weakly co-hopfian.

(5) Injective endomorphisms of $M_{R}$ map t-essential submodules to t-essential submodules.

(6) The inverse image of any submodule not in $Z_{2}(M)$ under any injective endomorphism of $M$ is not in $Z_{2}(M)$.

Proof:

(1) $\Rightarrow$ (2): Let $f: M \oplus N \rightarrow M$ be a monomorphism, $i: M \rightarrow M \oplus N$ is the canonical injection, then $f i$ is an injective endomorphism of $M$, hence $f i$ is t-essential. If now there exists a nonzero $K \leq f(N), K \cap Z_{2}(M)=0$, then we get a nonzero $K^{\prime}=K \cap f i(M)$, hence $K^{\prime} \leq f(M \oplus 0) \cap f(0 \oplus N)$, conversely. So $f(N) \leq Z_{2}(M)$; hence $N$ is $Z_{2}$-torsion.

(2) $\Rightarrow\left(\right.$ Q' $\left.^{\prime}\right)$ : this is trivial.

$\left(2^{\prime}\right) \Rightarrow(1)$ : Let $g$ be an injective endomorphism of $M$ without $t$-essential image. Then there exists a submodule $K$ not in $Z_{2}(M)$ satisfies $g(M) \oplus K \leq_{\text {tes }}$ $M$. But this yields a t-essential monomorphism $M \oplus K \rightarrow M$, contradicting (2'). Hence $g(M) \leq_{\text {tes }} M$.

(1) $\Rightarrow$ (3): Let $N$ be the injective endomorphism of $Z_{2}(M)$, then $N$ is nonsigular in $M$, so $N$ is weakly co-hopfian. For $N \oplus Z_{2}(M) \leq_{e} M$ we have $\left(\left(N \oplus Z_{2}(M)\right) / Z_{2}(M)\right) \leq_{e} M^{\prime}$. Then suppose $f$ be an injective endomorphism of $M^{\prime}, f\left(M^{\prime}\right)$ is not essential in $M^{\prime}$. So we have a $K \leq M, f(M) \oplus((K+$ $\left.Z_{2}(M)\right) / Z_{2}(M) \leq_{e} M$, thus $\left(K \oplus Z_{2}(M)\right) / Z_{2}(M) \cap\left(N \oplus Z_{2}(M) / Z_{2}(M) \neq 0\right.$. But $\left(N \oplus Z_{2}(M)\right) / Z_{2}(M)$ is weakly co-hopfian, then $f\left(\left(N \oplus Z_{2}(M)\right) / Z_{2}(M)\right) \cap$ $f\left(\left(K \oplus Z_{2}(M)\right) / Z_{2}(M)\right) \neq 0$, which is in $f\left(M^{\prime}\right) \cap\left(K \oplus Z_{2}(M)\right) / Z_{2}(M)$, contradicts. Then $M^{\prime}$ is weakly co-hopfian.

$(3) \Rightarrow(1):$ Suppose $g: M \rightarrow M$ be injective and $g(M)$ is not $t$-essential in $M$. Then we have an submodule $K$ of $M, g(M) \oplus K \leq_{e} M$ and $K$ is 
not in $Z_{2}(M)$. But $\left(g(M)+Z_{2}(M)\right) / Z_{2}(M) \oplus\left(K+Z_{2}(M)\right) / Z_{2}(M) \leq_{e} M^{\prime}$, contradicts the Dedekind finiteness of $M^{\prime}$. So $M_{R}$ is t-weakly co-hopfian.

$(1) \Rightarrow$ (4): Trivial.

$(4) \Rightarrow(1)$ : Assume that $K \leq_{\text {tes }} M, K$ is fully invariant and t-weakly co-hopfian. $g$ is an injective endomorphism of $M$, then $\left.g\right|_{K}$ is an injective endomorphism of $K$. So $g(K) \leq_{\text {tes }} K$, and $K \leq_{\text {tes }} M$, then we have $g(K) \leq_{\text {tes }}$ $M \Rightarrow g(M) \leq_{\text {tes }} M$.

$(1) \Rightarrow(5)$ : Suppose $A$ is a t-essential submodule of $M, f$ is an injective endomorphism of $M$. If there is an submodule $N$ in $M$ which is not in $Z_{2}(M)$, satisfies $f(A) \cap N \leq Z_{2}(M)$. Then we have $A \cap f^{-1}(N) \leq Z_{2}(M)$, but $f^{-1}(N)$ is not in $Z_{2}(M)$, contradicting that $A$ is t-essential. So $f(A)$ is t-essential too.

$(5) \Rightarrow$ (1): Trivial.

$(1) \Rightarrow(6)$ : Suppose $M_{R}$ submodule $N$ is not in $Z_{2}(M), f$ is an injective endomorphism of $M$, and $f(M) \cap N$ is not in $Z_{2}(M)$. Let $n$ be an element of $N$ not in $Z_{2}(M)$, and there exists an $m$ in $M$, such that $n=f(m)$. If $m$ is in $Z_{2}(M)$, we have $f(m)$ in $Z_{2}(M)$, contradicts. So $m$ is not in $Z_{2}(M)$.

$(6) \Rightarrow(1)$ : Assume an injective endomorphism $f$ of $M$ satisfies $f(M) \cap N \leq$ $Z_{2}(M)$ where $N$ is not in $Z_{2}(M)$, then we get $f^{-1}(f(M) \cap N) \leq f^{-1}\left(Z_{2}(M)\right) \leq$ $Z_{2}(M) \Rightarrow f^{-1}(N) \leq Z_{2}(M)$, contradicting $(6)$.

Corollary 2.3 If $M_{R}$ is t-weakly co-hopfian and $f$ is an injective endomorphism of $M$, then $N \leq_{\text {tes }} M$ if and only if $f(N) \leq_{\text {tes }} M$ if and only if $f^{-1}(N) \leq_{\text {tes }} M$.

Corollary 2.4 The following hold.

(1) A direct summand of a t-weakly co-hopfian module is t-weakly co-hopfian.

(2)A module is t-weakly co-hopfian whenever the injective envelope of $M / Z_{2}(M)$ is Dedekind finite.

Corollary 2.5 Let $M$ be quasi-injective.

(1)If $M$ is t-weakly co-hopfian so are any submodule and any finite direct sum of copies of $M$.

(2) Suppose $N$ is a fully invariant essential submodule of $M$. Then $N$ is $t$-weakly co-hopfian if and only if so is $M$. Moreover, $M$ is t-weakly co-hopfian if and only if so is $E(M)$.

Proof:

(1)For $M$ is t-weakly co-hopfian then we have $M^{\prime}=M / Z_{2}(M)$ is weakly co-hopfian. As in [2], $M^{\prime n}$ is weakly co-hopfian, so $M^{\prime n}=\bigoplus_{n} M / Z_{2}(M)=$ $\bigoplus_{n} M / \bigoplus_{n} Z_{2}(M)=\bigoplus_{n} M / Z_{2}\left(\bigoplus_{n} M\right)$. Then, due to theorem 1.1 (3), $t$-weakly co-hopfian property keeps the direct summand. Similarly, to prove that a submodule $A$ of $M$ is t-weakly co-hopfian, let $Z_{2}(M)=Z_{2}(A)+K$, then we notice that $A^{\prime}=A / Z_{2}(A) \cong(A+K) / Z_{2}(M) \leq M / Z_{2}(M)=M^{\prime}$, which means that $A^{\prime}$ is Dedekind finite. Thus $A$ is t-weakly co-hopfian. 
(2)The first section is obvious from theorem 1.1 and (1) above. For the second, we just notice that $M$ is a fully invariant t-essential submodule of $E(M)$ and then apply the first assertion.

\section{References}

[1] F. W. Anderson, K. R. Fuller, Rings and Categories of Modules(2nd), Springer-Verlag, New York, 1992.

[2] A. Haghany, M. R. Vedadi, Modules whose Injective Endomorphisms are Essential, Journal of Algebra, 1 (2001), 243, 765-779.

[3] A. Haghany, Sh. Asgari, T-Extending Modules and T-Baer Modules, communication in Algebra, 9 (2011),39 , 1605-1623.

[4] A. Haghany, Sh. Asgari, Y. Tolooei, T-Semisimple Modules and TSemisimple Rings, Communication in Algebra, 9 (2013),41, 1882-1902.

Received: January 5, 2014 